\title{
Introdução ao pensamento de Gustav Störring*1
}

\author{
German E. Berrios*2
}

Gustav Störring permanece sendo uma figura negligenciada apesar de sua contribuição aos campos da psiquiatria, filosofia e psicologia, nos quais realizou um trabalho pioneiro, particularmente na maneira como essas disciplinas se intercruzaram. Transpondo a segunda metade do século XIX e a primeira do século XX, Störring não apenas testemunhou mudanças no modo como a psicopatologia foi concebida, mas contribuiu ativamente para tal processo.

\section{O homem}

Gustav Wilhelm Störring nasceu em 24 de agosto de 1860 em Vörde, Vestefália, na Alemanha, de pais não acadêmicos (seu pai possuía uma pequena fundição). Foi muito estudioso e aprendeu Teologia e Filosofia na Universidade Halle, obtendo seu doutorado em Filosofia em 1890. Formou-se em Medicina em Berlim, sob a orientação de Emil du Bois Reymond, e obteve seu doutorado em 1894 (Steinberg and Kunstler, 2000).

\footnotetext{
*1 Tradução de Lumina Traduções.

*2 Universidade de Cambridge, Reino Unido.
} 
A formação combinada nas ciências humana e natural pode explicar muito dos interesses intelectuais posteriores de Störring. Em Leipzig, formou-se em Psiquiatria na clínica psiquiátrica de Hubertusburg, tendo trabalhado sob a orientação de Flechsig. Com o apoio de Wilhelm Wundt, em 1896 Störring obteve sua habilitação. Interrompeu, então, sua carreira acadêmica e, juntamente com sua esposa, Marie Bonacker (1866-1966), fundou e conduziu uma clínica psiquiátrica particular em Erdmanmshain, uma pequena cidade a sudeste de Leipzig.

De volta à vida universitária, entre 1902 e 1911, Störring manteve uma cátedra de filosofia em Zurique e, até 1914, um cargo similar em Strasburg. No mesmo ano, foi nomeado para uma cátedra universitária na Universidade de Bonn onde permaneceu até que se aposentou, em 1927. Störring faleceu em Göttingen, em 1 de dezembro de 1946, onde seu filho, Gustav Ernest Störring (também um eminente psiquiatra e filósofo) estava a trabalho no momento (Völkel, 1968).

\section{Sua obra}

Interessado em epistemologia desde muito cedo, a primeira obra de Störring sobre o tema foi "Mills Theorie über Ursprung des Vulgärglaubens an die Aussenwelt" (1889), que pode ser considerada uma precursora das visões atuais sobre a Psicologia Folk. A ela se seguiu "Zur Lehre von Einfluss der Gefühle auf die Vortellungen" (1896), expressando outro dos interesses intelectuais de Störring. Seu vasto pensamento epistemológico levou a "Die Erkenntnistheorie von Tetens (1901), Einführung in der Erkenntnistheorie” (1909); Erkenntnistheorie (1920) ${ }^{1}$ etc. Seu interesse em Psicologia e Psicopatologia é claramente mostrado em seus livros, como Vorlesungen über Psychopathologie in ihrer Bedeutung fur the normale Psychologie (1900), Psychologie (1920), Die Frage der geisteswissenschaflichen und verstehenden Psychologie (1928), Die Beziehungen zwischen Psychologie und philosophischer Ethik (1932) etc. Por fim, tendo convicção evangélica,

\footnotetext{
${ }^{1}$ Considerou-se que este livro, e o ensinamento geral de Störring, influenciou Moritz Schlick durante o período em que esteve na Universidade de Zurique. A visão de Störring da filosofia como 'WissenschaftsLehre', suas visões sobre o conceito de espaço e sua crítica do positivismo e do neokantinismo estão claramente refletidas no Allgemeine Erkenntnislehre (1918) de Schlick. Schlick tornou-se chefe do Círculo de Viena (Neuber, 2007).
} 


\section{CLÁSSICOS DA PSICOPATOLOGIA}

Störring também tinha interesses em teologia: Die Frage der Wahrheit der christlichen Religion (1920) (Ziegenfuss and Jung, 1950).

\section{O texto}

O texto a seguir é uma tradução da primeira palestra sobre Vorlesungen über Psychopathologie in ihrer Bedeutung fur the normale Psychologie (1900) de Störring, que muitos consideram seu livro mais inovador. Dedicado a seu professor Wundt, ele se baseou em uma série de 25 palestras ministradas enquanto era um palestrante externo (Privatdozent) em Filosofia, na Universidade de Leipzig. O livro recebeu uma boa crítica no mundo literário da época (Anônimo, 1900; Sidis, 1908).

O livro é importante por três razões: 1) o uso particular do termo Psychopathologie; 2) conectar sintomas mentais à Psicologia normal; e 3) a discussão da estrutura epistemológica dos sintomas mentais.

\section{1) O termo Psychopathologie}

Embora o termo composto Psycho-pathologie tenha primeiro aparecido em 1845 em um livro de Feuchtersleben (1845), a palavra Psicopatologia (sem o hífen) foi formada quando a palavra em alemão foi transliterada para a versão inglesa do seu livro (1847). Levou muito tempo para o termo ser incorporado à linguagem médica e psicológica. Em alemão, ele foi primeiro utilizado como um título em Allgemeine Psychopathologie. Zur Einführung in das Studium der Geistesstörungen (1878), escrito por Hermann Emminghaus, que então trabalhava em Würzburg sob a orientação de Franz von Rinecker, a quem ele dedicou o livro. Karl Jaspers (1913) pegou emprestado de Emminghaus o nome para seu próprio livro, cuja primeira edição foi publicada em 1913. Uma versão em francês do termo apareceu na edição de 1912 (Marie, 1912). Em 1911, o grande livro sobre sintomas mentais de Philip Chaslin (1912) ainda mostrava o velho nome francês Sémiologie.

$\mathrm{O}$ uso feito por Emminhaus do termo Psychopathologie é quase equivalente a Psychiatrie, de tal modo que, além de sintomas mentais, ele também aborda transtornos mentais, etiologia, predisposição, diagnóstico e prognóstico dos mesmos. Em Störring (assim como posteriormente em Chaslin e Jaspers), ocorre o contrário, isto é, há muito mais ênfase nos próprios sintomas mentais, na dinâmica de sua construção e no seu papel na formação histórica das doenças mentais. 


\section{2) Os vinculos entre sintomas mentais e psicologia normal}

A segunda metade do século XIX testemunhou um importante debate sobre se o estudo dos sintomas mentais deveria ser realizado por uma disciplina especial chamada 'Psicopatologia' ou se ele deveria ser parte da psicologia normal como 'Psicologia Patológica'. O debate não era sobre os termos em si, mas sobre a etiologia e a epistemologia dos sintomas mentais (Berrios, 1988). Até a década de 1870, a Psicologia se comportou como uma disciplina filosófica e conceitual (o que Störring denomina 'psicologia metafísica') e, sendo assim, tinha pouca pretensão de lidar com sintomas mentais que permaneceram claramente dentro do território do Alienismo. ${ }^{2}$ Embora os alienistas tenham usado os termos e conceitos psicológicos para mapear os sintomas mentais, a visão geral era de que estes eram significantes e blocos de construção das doenças mentais e, sendo assim, claramente localizados dentro do território da medicina (Berrios, 1996).

Durante a segunda metade do século XIX, a visão de que os sintomas mentais poderiam ser mais bem estudados, não de maneira independente, mas como desvios das conhecidas funções psicológicas, foi incentivada

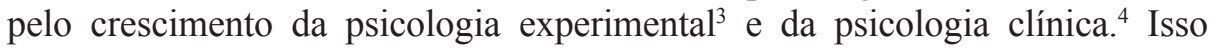
representou um desafio da Psicologia para o Alienismo e um debate que se seguiu sobre os limites do território ao qual os sintomas mentais pertenciam. As visões de Störring são representativas de um terceiro grupo de pensadores na área. Eles não eram psicólogos nem alienistas convictos, mas pensadores vindos da filosofia e com fortes interesses filosóficos (como Morselli, Chaslin, Ziehen e Jaspers). Esses homens adotaram uma visão conceitual e objetiva da disputa e acreditavam que uma estreita associação (não uma fusão) entre a

${ }^{2} \mathrm{O}$ Alienismo (agora denominado Psiquiatria) dá nome à disciplina e à profissão, construídas no começo do século XIX, para lidar com a loucura. Na maioria dos países europeus, foi rapidamente profissionalizado como um setor, ciência, práxis etc. e desenvolveu ritos de passagem, exames, textos e periódico. Além disso, reivindicou o direito de gnovos espaços físicos que mantinham os mentalmente afligidos (os hospícios).

${ }^{3}$ A literatura sobre a história do desenvolvimento da psicologia experimental do século XIX é agora vasta e uma historiografia convencional (Boring, 1950) e uma revisionista (Danziger, 1990; Richards, 2010) podem ser diferenciadas.

${ }^{4}$ Ribot, Janet, Sully, Charcot, Dumas, Wundt, Kraepelin, Preyer etc. podem ser lembrados entre aqueles que fizeram as primeiras pesquisas em psicologia clínica (Routh, 2011). 


\section{CLÁSSICOS DA PSICOPATOLOGIA}

psicopatologia convencional e a psicologia clínica poderia beneficiar ambas as partes e a compreensão dos sintomas mentais.

\section{3) Interesse na estrutura epistemológica dos sintomas mentais}

Nas mãos dos alienistas, pressupunha-se que os sintomas mentais tivessem a mesma estrutura e função dos sintomas físicos e que realizassem o mesmo trabalho. De acordo com a Escola de Medicina de Paris (Ackerknecht, 1967), os sintomas eram significantes na medida em que na mente do médico treinado eles poderiam ser vinculados a doenças específicas (Landré-Beauvais, 1818). Sua etiologia era, portanto, a mesma da doença que elas significavam ou representavam. Rápido o bastante, e após a introdução da subjetividade ${ }^{5}$ como uma fonte adicional de informações em medicina, sinais e sintomas foram diferenciados: os primeiros apresentando a si próprios diretamente ao olho empírico do homem médico e os últimos estando ocultos na subjetividade do queixoso (e, assim, não diretamente presente na observação empírica).

Esse modelo mecanicista foi adotado pelos alienistas e, portanto, havia pouco interesse em fazer perguntas adicionais sobre sua relevância para a loucura. Na segunda metade do século XIX, entretanto, os psicólogos e os filósofos começaram a fazer tais perguntas. A razão para essa inquietude epistemológica era que, embora a loucura parecesse apresentar ambos os sinais (transtornos do movimento, febre, descolorações da pele etc.) e os sintomas (alucinações, delírios, obsessões, tristeza etc.), parecia claro que os últimos eram predominantes e eram muito mais específicos e significativos para a loucura do que os sinais físicos não específicos.

Perto do fim do século XIX, a psicologia clínica poderia estar em uma posição a oferecer uma explicação para a predominância dos sintomas mentais em relação aos sinais, mas eles também estavam ávidos, como uma nova ciência experimental, em desenvolver credenciais neurocientíficas. ${ }^{6}$ Foi deixado aos filósofos ${ }^{7}$ e àqueles interessados em hermenêutica fazer as

${ }^{5}$ Estas alterações epistemológicas são paralelas à introdução e aceitação do conceito de introspecção (Danziger, 1980).

${ }^{6}$ Este fenômeno pode ainda ser observado hoje em dia em alguns países (por exemplo, no Reino Unido) onde há uma tendência entre psicólogos de favorecer narrativas neurocientíficas e a "neuropsicologia"; de fato, muitos estão muito mais interessados em imagens neurais e na parafernália da pesquisa neurológica do que em desenvolver narrativas psicológicas da loucura.

${ }^{7}$ Lotze (Berrios, 2005) e Dilthey (Caparrós, 1986) são exemplos notáveis dessa posição. 
perguntas mais difíceis sobre se os "sintomas mentais" eram de fato diferentes na estrutura e função dos sintomas físicos. A revolução hermenêutica obviamente culminou em Freud e seus seguidores que introduziram um modelo interpretativo verídico para os sintomas mentais.

Störring, talvez por conta de seus interesses epistemológicos, foi um dos primeiros expoentes deste movimento na Alemanha. Sua visão não era apenas a de que alguns sintomas mentais pudessem, de fato, ser desvios da função psicológica normal (tal como a ansiedade pudesse ser um exagero do medo), mas de que, em geral, o estudo dos transtornos psicopatológicos pudesse ajudar a jogar uma luz sobre a epistemologia do comportamento normal. Karl Jaspers desenvolveu essas visões vinculando suas próprias interpretações do neokantianismo à noção da "compreensão" de Dilthey e Windelband (Jaspers, 2013). Por outro lado, a verdadeira influência da fenomenologia husserliana sobre o trabalho do filósofo Heidelberg é menos clara e requer mais pesquisa (Berrios, 1992).

O fascinante insight de Störring, posteriormente desenvolvido por Jaspers, é que o modelo anatomofisiológico da doença, que funciona tão bem em medicina, não é muito útil na psiquiatria porque a loucura inclui um "fator mental" ('psychischen Faktor') que escapa da captura pela narrativa neurocientífica e cujo estudo, não obstante, é essencial para compreender a loucura: Andere Autoren, die ebenfalls die anatomisch-physiologische Betrachtungsweise überschätzen, gehen nicht so weit, dass sie den psychischen Faktor völlig ignorieren... (p. 15) ("outros escritores que, do mesmo modo, superestimam a relevância do ponto de vista anatomofisiológico não vão tão longe a ponto de ignorar o fator mental...”). Sendo medicamente qualificado, foi talvez compreensível que Störring houvesse adotado uma posição intermediária entre a neurobiologia e a psicologia.

\section{Referências}

Ackerknecht, E. (1967). Medicine at the Paris Hospital 1794-1848. Baltimore: Johns Hopkins Press.

Anonymous (1900). Vorlesungen über Psychopathologie. The Monist. 11, 136-138.

Berrios, G.E. (1988). Historical Background to Abnormal Psychology. E. Miller \& P.J. Cooper (Eds.), Adult Abnormal Psychology (pp. 26-51). Edinburgh: Churchill and Livingstone. 


\section{CLÁSSICOS DA PSICOPATOLOGIA}

Berrios, G.E. (1992). Phenomenology, psychopathology and Jaspers: a conceptual history. History of Psychiatry 3, 303-327.

Berrios, G.E. (1996). The History of Mental Symptoms. Cambridge: Cambridge University Press.

Berrios, G.E. (2005). Lotze and his "Medicinische Psychologie oder Physiologie der Seele". History of Psychiatry, 16, 117-127.

Boring, E.G. (1950). A History of Experimental Psychology. New York: AppletonCentury-Crofts.

Caparrós, A.H. (1986). Ebbinghaus. Un funcionalista investigador tipo dominio. Barcelona: Publicacions Universitat de Barcelona.

Chaslin, P. (1912). Eléments de Sémiologie et de Clinique Mentale. Paris: Asselin et Houzeau.

Danziger, K. (1980). The History of Introspection reconsidered. Journal of the History of the Behavioural Sciences 16, 241-262.

Danziger, K. (1990). Constructing the subject: historical origins of psychological research. Cambridge: Cambridge University Press.

Emminghaus, H. (1878) Allgemeine Psychopathologie. Leipzig: FCW Vogel.

Feuchtersleben, E. von (1845). Lehrbuch der ärztlichen Seelenkunde. Wien: Carl Gerold.

Feuchtersleben, E. von (1847). The Principles of Medical Psychology. Translated by H.E. Evans \& B.G. Babington. London: Printed for the Sydenham Society.

Jaspers, K. (1913). Allgemeine Psychopathologie. Heidelberg: Springer.

Landré-Beauvais A.J. (1818). Séméiotique ou traité des signes des maladies. $3^{\text {rd }}$ Edition. Paris: Brosson.

Marie, A. (Ed.) (1911-1912). Traité International de Psychologie Pathologique. 4 vols, Paris: Alcan.

Neuber, M. (2007). "Schlick, Friedrich Albert Moritz". Neue Deutsche Biographie, 23, 78-80.

Richards, G. (2010). Putting Psychology in its Place: critical historical perspectives. $3^{\text {rd }}$ Edition. London: Routledge.

Routh, D.K. (2011). A History of Clinical Psychology. In D.H. Barlow (Ed.), Oxford Handbook of Clinical Psychology (pp. 23-33). Oxford, Oxford University Press.

Sidis, B. (1908). Review of Mental Pathology and its relation to normal psychology. Journal of Philosophy, Psychology and Scientific Methods, 5, 382-389.

Steinberg, H. and Künstler, U. (2000). Vor 100 Jahren erschienen die "Vorlesungen über Psychopathologie..." von Gustav Wilhelm Störring. Fortschritte Neurologie Psychiatrie, 68, 243-249.

Schlick, M. (1918). Allgemeine Erkenntnislehre. Berlin: Springer 
Völkel, H. (1968). G E Störring zum 65. Geburtstag. Zeitschrift für Psychosomatische Medizin und Psychoanalyse, 14: 77-78.

Ziegenfuss, W. \& Jung, G. (1950). Gustav Störring. Philosophen-Lexicon (V, 2, p. 644-645). Berlin, Walter de Gruyter.

Citação/Citation: Berrios, G.E. (2016, junho). Introdução ao pensamento de Gustav Störring. Revista Latinoamericana de Psicopatologia Fundamental, 19(2), 303-310.

Editor do artigo/Editor: Prof. Dr. German E. Berrios Recebido/Received: 6.6.2016 / 6.6.2016 Aceito/Accepted: 10.6.2016 / 6.10.2016

Copyright: (C) 2009 Associação Universitária de Pesquisa em Psicopatologia Fundamental/ University Association for Research in Fundamental Psychopathology. Este é um artigo de livre acesso, que permite uso irrestrito, distribuição e reprodução em qualquer meio, desde que o autor e a fonte sejam citados / This is an open-access article, which permits unrestricted use, distribution, and reproduction in any medium, provided the original authors and sources are credited.

\section{German E. Berrios}

Médico e filósofo pela Universidad Nacional de San Marcos, Lima, Peru; Psiquiatra; Neurologista; Psicólogo; Filósofo; Historiador e Filósofo da ciência (Oxford University, England); Professor de Neuropsiquiatria e de Epistemologia da Psiquiatria (University of Cambridge, England), desde 1976; Neuropsiquiatra e Chefe do Departamento de Neuropsiquiatria do Hospital Addenbrooke, University of Cambridge, por 32 anos; Coordenador do Comitê de Ética em Pesquisa com Humanos na mesma universidade, por vinte anos; Editor Responsável de History of Psychiatry; Autor de 14 livros, incluindo The History of Mental Symptoms, Descriptive Psychopathology since 19th Century (Prêmio Nacional BMA, 1997), A History of Clinical Psychiatry (com Roy Porter), e Delirio (com F. Fuentenebro) e mais de 400 artigos e capítulos de livros; Membro do Royal College of Psychiatrists, da Associação Britânica de Psicologia e da Academia Britânica de Ciências Médicas; Membro Vitalício do Robinson College, Cambridge; doutor Honoris Causa da Universidade de Heidelberg (Alemanha), da Universidade Nacional Mayor de San Marcos (Peru) e da Universidad Autónoma de Barcelona (Espanha); Grão Oficial da Ordem del Sol (Condecoração do Governo Peruano, 2007); prêmio Ramon y Cajal 2008 concedido pela Asociación Internacional de Neuropsiquiatria.

University of Cambridge

Box 189, Hills Road

Cambridge,

UK CB2 2QQ

e-mail: geb11@cam.ac.uk

This is an open-access article, which permits unrestricted use, distribution, and reproduction in any medium for non-commercial purposes provided the original authors and sources are credited. 